\section{A report from the first regional pain medicine symposia in East, Central and Southern African region. (ECSA-R)-“The Rhino Model in Pain \\ Education in Africa."}

Thikra Sharif, John Bugo

Aga Khan University Hospital, Anaesthesiology Nairobi, Kenya

\section{DOI: http://dx.doi.org/10.4314/ahs.v15i2.17}

Introduction

be based on the recent academic developments in the The 1st Regional pain medicine symposia were held knowledge of pain medicine, and should be delivered between the 14th and 18th of November 2011 in 3 by qualified pain and neuroscience team of specialists. cities (Nairobi, Mombasa and Dar-es-salaam), across 2

International Association for the Study of Pain (IASP), The Rhino model in pain education in sub-Saharan Afthrough the IASP developing country education grant rica is an academic and clinical program model, whose for 2011 and The Aor Khan University Hospital Nairo- main objectives are to introduce and spread the theobi (AKUHN). It was organized by faculty from the Pain retical and the clinical concept of pain medicine, and Management Unit (PMU) AKUHN. The Pain medicine to introduce pathways in building a foundation for pain educators were from PMU, AKUHN and from Special- management services in the East, Central and Southists in Pain International Network (SPIN).

The aims of the symposia were to introduce the Rhino model concept, the theoretical and clinical concepts of pain medicine, as well as create awareness and stimulate an interest in pain medicine education and pain research in ECSA region. At each of the 3 different locations, the same topics and workshops were presented by the speakers, thus ensuring uniformity of the message delivered.

\section{Definition and concept of the Rhino model in pain}

\section{education in Africa}

Definition

The Rhino model is an academic and clinical multidisciplinary Neuroscience based pain management program model, that was developed by Dr.Thikra Sharif in 1998. It explores and advocates the depth of the concept of pain medicine. This requires education across the board for undergraduate and postgraduate medical fraternity, nurses and doctors. Such education, of necessity mus

\section{Corresponding author}

Thikra Sharif,

Aga Khan University Hospital,

Pain Management/Anaesthesiology

Email: pain.management@aku.edu

Objectives of the Rhino mode

To introduce the concept and the 7 stages of development of the Rhino model in pain medicine education in CSA region.

To identify at least one person in every location, a "junor rhino" and help him/her to acquire an accredited pain medicine specialist qualification. The "junior rhino" will then facilitate the development of a unified training program in all regions (North Africa, West Aftica, ECSA, and South Africa).

To convince the relevant authorities that building a strong foundation (based on the Rhino model concept) is a reality even in low resources countries; and, being in a resource challenged area does not mean a strong foundation based on neuroscience cannot be established.

To promote wider coverage of pain management services by providing training and service programs through countries, Kenya and Tanzania. It was funded by the Concept
African Health Sciences Vol 15 Issue 2, June 2015 African region.

\section{The Rhino model stages of development}

Stage 1: Identify a renowned institution (institution A) which has the authority to train and award qualifications in pain medicine. This institution will graduate the first pain medicine specialist. "The first Rhino".

Stage 2: Identify an authority in pain medicine, in institution $\mathrm{A}$, who will be able to deliver administrative and clinical experience in pain medicine to the first Rhino.

Stage 3: The first Rhino will then acquire administrative and clinical knowledge in pain medicine through the academic program in institution $\mathrm{A}$

Stage 4: The first Rhino will replicate the acquired academic and clinical model of institution $\mathrm{A}$, and transfe it to a new recipient location, example the Aga Khan University Hospital Nairobi.

Stage 5: The first Rhino will revise the model of institution A to suit the financial, administrative and environmental circumstances of the new location, and follow the protocols of institution A.

Stage 6: The first Rhino will incorporate the program preferably in a university set up. The objectives of incorporating such a programme in a university curriculum are as follows:

. Universities are more likely to have an academically oriented multidisciplinary team.

b. Universities have better financial back up and support for academic programs.

c. Universities will enable the first Rhino to target the young residents who will be the future ambassadors in pain medicine in the region.

d. Universities will enable the first Rhino to incorporate an academic program in pain medicine within the university post graduate training, which will then ensure a standardized knowledge and a standardized clinical training in pain medicine across all specialties.

e. Research - the first Rhino will enhance knowledge in pain medicine among residents to stimulate their ideas for pain research proposal. Research can never be initiated in any field without prior knowledge about the subject.

\section{Stage 7: The future challenges:}

a. To promote wider coverage; providing training and service programs through outreach courses and missions

b. Through these courses and missions the first Rhino will be able to identify at least one "junior rhino" in every location north-, west- and ECSA, who will be willing to follow the rhino tracks in pain education. Therefore within 5-6 years we should have qualified junior Rhinos that will be ready to continue the mission.

Proposing continental collaboration and unifying training programs in all regions- North African, West African, ECSA, and South Africa, and working towards a joint accreditation and award of a pain management specialist degree.

Figure 1: The Rhino model in Pain medicine education in Africa

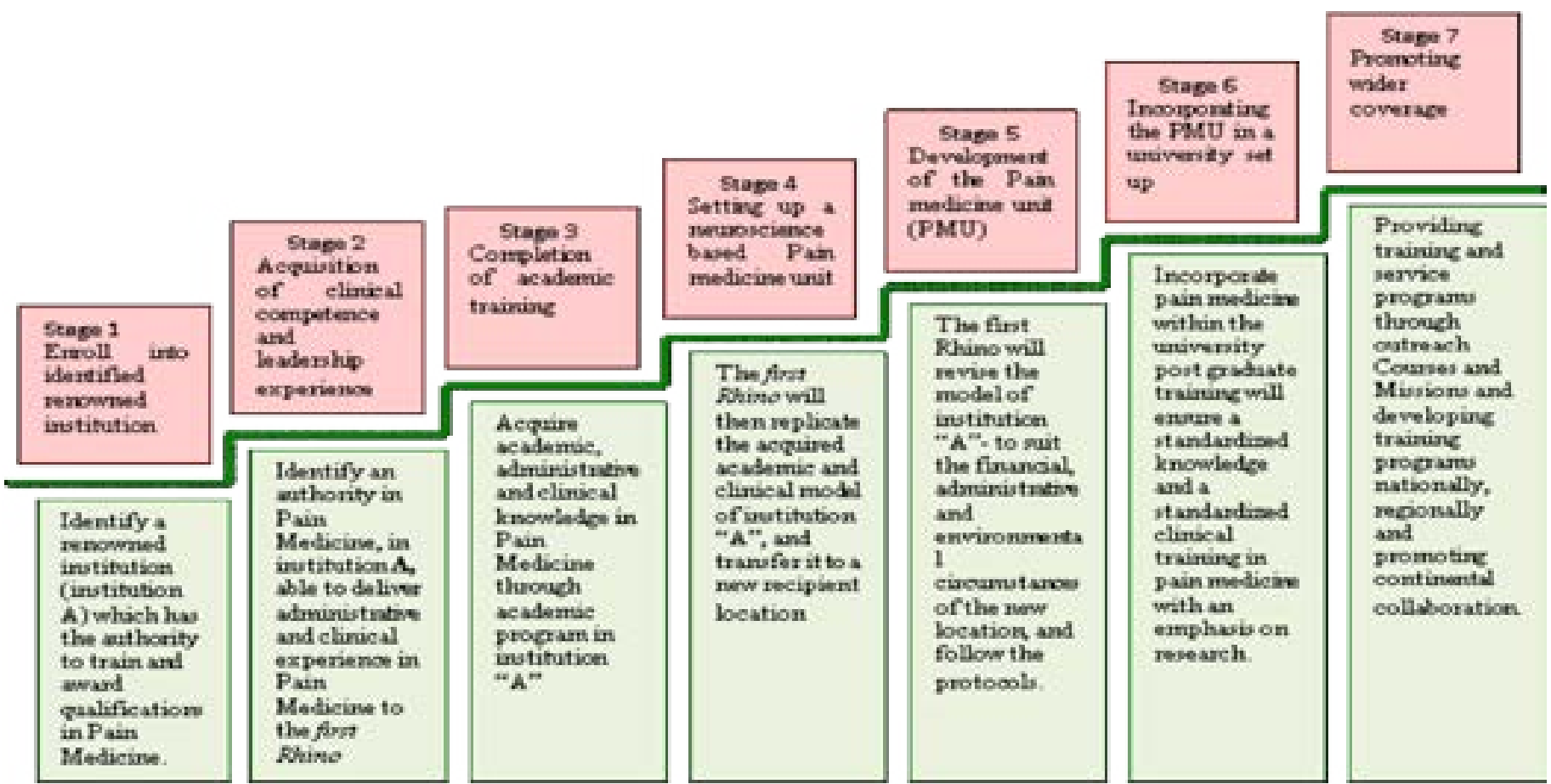

African Health Sciences Vol 15 Issue 2, June 2015 
Symposium report

The presentations on the different aspects of pain medicine were made by the following faculty:

Dr. Thikra Sharif - Consultant in anaesthesia and pain medicine specialist, AKUHN

Dr. Gilbert Mwaka - Consultant in anaesthesia and Concept of pain (Thikra Sharif)

pain management fellow, AKUHN/ Sydney University. This lecture gave the definition and general considerDr Brigitta Brandner- Consultant in anaesthesia and ations of acute pain and chronic pain. It was stressed pain management UCLH-NHS Foundation Trust UK. that improper management of acute pain can cause the Dr. John Lee - Consultant in anaesthesia and pain acute pain to persist and progress to chronic pain. Pain management, the National Hospital for Neurology and was classified into nociceptive, neuropathic and psychoNeurosurgery, UK, Queens Square.

Professor Lesley Bromley - Consultant in anaesthesia \& pain management UCLH-NHS Foundation Trust UK. Mr. M.M. Qureshi FRCS - Consultant Neurosurgeon AKUHN and Kenyatta National Hospital \& Section. Dr. Vitalis Mung'ayi - Consultant in anaesthesia and critical care specialist, AKUHN

Dr. Dorothy Kamya - Consultant in anaesthesia and medical education, AKUHN.

A summary of the major points discussed in each presentation

Challenges of setting up a multidisciplinary neuroscience based pain management Unit - Rhino

Model in Pain education in Africa (Thikra Sharif) This lecture defined the concept of the Rhino model in pain management and in pain education in Africa, emphasizing on its role in introducing and spreading the theoretical and the clinical concept of pain medicine, and in introducing pathways towards building a foundation for pain management services in the region that is based on a firm pillar of neuroscience. The presentation also highlighted the necessity of introducing the biopsychosocial model and the multidisciplinary concept in diagnosis, treatment and management of painful syndromes. The presentation also stressed the need for pain medicine research in sub-Saharan Africa. The burden of pain management in sub-Saharan Africa was outlined as well as the current status of specialized pain management services in the region.

\section{Assessment and measurement of pain (Thikr} Sharif)

This presentation described comprehensively the difference between the assessment and measurement

of pain, the value of comprehensive assessment and the necessity of a comprehensive clinical interview

in pain assessment. It stressed on the value of the diagnostic strategy in pain management and outlined the vival, optimizing comfort, optimizing function and relieving pain were emphasized. The etiology of pain in cancer patients, the role of palliative care, the sionificance of the WHO pain relief ladder, the analgesic techniques available as well as end of life care were al discussed.

\section{Neurobiology of pain (Brigitta Brandner)}

The concepts of autosensitization and wind-up, peripheral and central sensitization and persistent pathological pain were introduced (pain, plasticity and gain). Mechanisms of peripheral and central sensitization and activation of NMDA receptor, NK-1 tachykinin receptor and AMPA receptor were elucidated. A discussion on how the memory of pain can be more damaging than its initial experience was held. Pathways of pain modulation as well as genetically "built in" brain processes accounting for the phantom limb phenomenon was discussed. The concept of the body-self neuromatrix was also briefly discussed.

The Biopsychosocial model (John Lee)

This lecture gave a definition of the Biopsychosocial model in pain management and described its components. The biological component relates to nociception the psychological component is the influence of anxiety and/or depression on the perception of pain and this is why a psychiatrist/psychologist is an important member of a multidisciplinary team. In many patients social and environmental factors can be crucial in coping with pain. The presenter highlighted the importance of utilizing this model in the diagnosis and treatment of $\mathrm{pa}$ tients with painful syndromes.

\section{Pediatric pain (Lesley Bromley)}

This presentation emphasized that pain in children is real and should be treated. It demonstrated that assessment of pain is possible in preverbal children and the WHO pain relief ladder works. The rules of pediatric analgesia were discussed. These were: always give analgesics by weight, by mouth whenever possible and by the clock (always regular never Pro re nata (p.r.n).

\section{Analgesic pharmacology (Lesley Bromley)}

This lecture emphasized the importance of a multimodal approach to analgesic treatment. It also put emphasis on paracetamol forming the backbone of the pain medications and careful use of NSAIDs. The lecture stressed that opioids/opiates are effective in the correct dose and their mechanisms of action are now better the role of genetics in analgesic pharmacology was highlighted.

Spinal interventions for chronic low back pain (Mahmood Qureshi)

The presentation discussed the epidemiology, associated risk factors and the burden of chronic low back

pain to society. It outlined the non-operative minimally invasive intervention procedures available for treatment of chronic low back pain, providing a brief outline of the procedures, their indications and individual ole in management. The interventions available at the AKUHN were highlighted.

Trigeminal neuralgia: Current concepts of management (Mahmood Qureshi)

The presentation discussed the anatomical basis and the aetiological factors underlying Trigeminal Neuralgia (TGN). A historical perspective and the various therapeutic options were outlined. The role of pharmacotherapy, injection procedures, thermo-coagulation and micro-vascular surgical decompression was discussed. The current technique utilized at AKUHN, of Balloon compression of the trigeminal nerve at the foramen ovale was highlighted.

Training doctors and nurses in pain medicine-East Africa (Vitalis Mung'ayi and Dorothy Kamya)

These talks highlighted the emerging challenge of educating health professionals in the concept of pain and pain management in the ECSA region. Beginning with an emphasis on the physical, social, economic and psychological burden of pain, the speakers pointed out the lack of definitive data on the problem of pain and the lack of trained personnel for relieving patients' suffering. Across the ECSA region, there is only one specialist run pain management unit, in contrast to the UK for example, where there are 260 units. Drawing attention to the need for a multidisciplinary approach for management of pain, it was stated clearly that effective training programs must be multi-professional; encompassing physician, nurse, physiotherapist, psychologist and patient education.

Summary of feedback forms from the attendants.

All comments from the audience at the three locations of the symposia were reviewed by the faculty at the end of the symposia. The feedback from participants 
Qurding the symposia and workshop was as follows. Question 1: Invited speakers' feedback. What did you think of them?

The quality of the invited speakers was scored highl with the majority (98\%) complementing the knowledge and wide scope covered by the speaker.

Question 2: Workshops' feedback: what did you think of them?

The majority (88\%) recommended a longer workshop duration of 2-3 days. With regards to material covered and organization and presentations, (95\%) were satisfied.

Question 3: What did you hope to gain from the pain symposium?

Over $95 \%$ of the audience reported that the objectives of the workshops were achieved, these included information on the Rhino model in pain education program in ECSA, learning new modalities of pain management apart from analgesics, learning how to assess pain, and improve knowledge on acute and chronic pain management.

\section{Question 4: What was good about the pain sympo-} sium?

$98 \%$ of the audience reported that the symposium was valuable as it was the first such endeavor. The existence of pain medicine in the region and the pain management unit at the Aga Khan university hospital was available to offer a training program in pain medicine to the region. They also recognized the Rhino Model as a valuable concept.

Question 5: what further action has the meeting prompted you to do?

Several participants sought to be enrolled for furthe

education in pain medicine as the "Junior Rhinos", seek

to develop pain management services and research in their countries.

Question 6: what are your suggestions for future conferences?

The majority (95\%) of the participants requested for an annual pain medicine symposium.

Question 7: do you have any other comments/observations?

The majority of the participants requested to avail op- portunities for residents and doctors to have elective posting at the pain management unit at the Aga $\mathrm{kl}$ the provite short courses with certificates on pain medicine training

\section{Conclusion}

While the main objective of the symposia was not to identify a "junior rhino", but rather the need to sensitize each region to the need for one, who will later develop a pain education program using the Rhino model in pain education in Africa. The faculty however was able to identify at least one "Rhino" in every location and guide them to follow the rhino track through acquiring accredited pain management specialist qualification. Follow up on the junior rhinos that were identified, would be through contact with the local coordinator or ead of department with the following avenues of progression available to them: a) A site where they can be exposed to a working pain management unit b) Link up with fellowship programs internationally through links with SPIN international in the UK. Several authorities in attendance during the symposia were addressed. These were people in positions of influence within the healthcare system in their respective countries, including consultants and health ministry officials. A concerted effort had been made to invite the policy makers, health ministry officials, senior consultants in the medical field and hospital administrators to attend the symposia, and the response was positive. Hospital administrators wrote their commitment to fellowship training, committed space and finances for setting up acute pain services, and serious discussions were held with SPIN international officials to set up protocols for the acute pain management programs in their respective hospital wards. A total of 600 health care professionals including doctors and nurses attended the 3 symposia and workshops.

As a follow up from the first regional pain symposia the plan was set for the second regional pain medicine symposia in ECSA region to be held in 2012 in Kenya, Uganda and Rwanda. The plan was successfully achieved, and the second regional pain medicine symposium in ECSA region was held on the 17th-18th November Kigali, Rwanda and in Nairobi, Kenya 21st22nd November 2012. This was followed with the third regional pain medicine symposium in ECSA region was held again on the 17th-21st September 2013 in Kigali, Rwanda and in Nairobi, Kenya.
The first regional pain medicine symposia in ECSA re- specialists in pain medicine. The course also delivered gion held in 3 cities in 2 countries between 14th and a message to the relevant authorities, that building a 18th November 2011 was successful in promoting pain strong foundation (based on the Rhino model concept) medicine and pain education in Africa. It provided a in pain medicine and pain education is a reality even valuable way of introducing the theoretical and the clin- in low resource countries. As a follow up to the first ical concepts of pain medicine delivered by qualified regional pain symposia. 